Cláudia Giglio de Oliveira Gonçalves ${ }^{\mathrm{a}}$ iD https://orcid.org/0000-0001-8316-1392

Francisca Pinheiro Fontoura ${ }^{a}$ iD https://orcid.org/0000-0003-3778-1528

a Programa de Mestrado e Doutorado em Distúrbios da Comunicação, Universidade Tuiuti do Paraná, Curitiba, PR, Brasil.

Contato:

Cláudia Giglio de Oliveira Gonçalves

E-mail:

claudia.goncalves@utp.br

Os autores declaram que o estudo não foi subvencionado e que não há conflitos de interesses.

Os autores informam que o trabalho não foi baseado em dissertação ou tese e não foi apresentado em eventos científicos.

\section{Intervenções educativas voltadas à prevenção de perda auditiva no trabalho: uma revisão integrativa}

\author{
Educational interventions on prevention of hearing loss at work: \\ an integrative review
}

\section{Resumo}

Objetivo: analisar a produção científica sobre intervenções educativas desenvolvidas para a prevenção auditiva de trabalhadores expostos ao ruído. Método: revisão integrativa, descritiva e analítica de produções científicas publicadas no período 2005-2016, em idioma inglês e português, disponíveis nas bases de dados LILACS, MEDLINE, PubMed, SciELO e Google Scholar. Resultados: inicialmente foram identificados 137 artigos e após aplicação dos critérios de inclusão e exclusão, 16 artigos foram selecionados (9 internacionais, 7 nacionais) para análise. As categorias profissionais nos estudos foram: mineiros, operários da construção civil, bombeiros, operários de empresa de equipamentos eletrônicos, agricultores, trabalhadores de frigorífico, teleoperadores, trabalhadores de universidade, pescadores industriais e trabalhadores de empresa alimentícia. A maioria dos estudos visava a mudança de atitude em relação aos protetores auriculares. Os estudos internacionais basearam-se em teoria comportamental e pretendiam aumentar a intenção de uso e a utilização de protetores auriculares; apenas um deles visou a redução do nível de ruído no ambiente de trabalho. Dos estudos nacionais, 3 utilizaram abordagens comportamentais e 4 desenvolveram ações educativas, mas somente 1 referiu metodologia participativa. Conclusão: intervenções educativas focadas apenas na utilização de protetores auriculares mostraram-se insuficientes, indicando a necessidade de ações educativas mais abrangentes e participativas, associadas a medidas de controle ambiental.

Palavras-chave: saúde do trabalhador; ruído ocupacional; prevenção; perda auditiva induzida por ruído; educação em saúde.

\begin{abstract}
Objective: to analyze the scientific production about educational interventions aimed at prevention of Noise-Induced Hearing Loss at work. Method: descriptive and analytical integrative review of scientific literature published between 20052016, in English and Portuguese, available in LILACS, MEDLINE, SciELO, PubMed and Google Scholar. Results: initially, 137 articles were identified; after application of inclusion and exclusion criteria, 16 articles (9 international, 7 national) were selected for analysis. The occupational categories included in the studies were: miners, firefighters, telemarketers, farmers, industrial fishermen, and workers of universities, electronic equipment industry, construction, meat processing, and food companies. Most studies aimed at changing the attitude towards hearing protectors. The international investigations were based on behavioral approaches and had the purpose of increasing intent of use and use of hearing protectors; only one of them was aimed at reducing noise level in the work environment. Among the national studies, three adopted behavioral approaches, and four developed educational activities, but only one mentioned a participatory methodology. Conclusion: educational interventions, which were only focused on the use of hearing protectors, were insufficient, indicating that they must be more comprehensive and participatory, as well as associated with environmental control measures.
\end{abstract}

Keywords: occupational health; occupational noise; prevention; noise-induced hearing loss; health education. 


\section{Introdução}

O ruído presente nos ambientes de trabalho é considerado um agente de risco nocivo à saúde dos trabalhadores, podendo causar doenças ocupacionais, como a perda auditiva induzida por ruído (PAIR). A exposição prolongada a níveis de ruído acima de $80 \mathrm{~dB}(\mathrm{~A})$ traz grande risco para a audição e a saúde em geral, a depender da intensidade e do tempo de exposição. O comprometimento na audição é permanente e, atualmente, não há qualquer tratamento efetivo para a regeneração da cóclea, principal local de lesão por ruído elevado ${ }^{1}$.

Dados do Departamento de Trabalho dos Estados Unidos, Administração de Segurança e Saúde no Trabalho (OSHA - Occupational Safety and Health Administration) ${ }^{2}$ estimam em 22 milhões os trabalhadores americanos expostos no trabalho a níveis de ruído potencialmente prejudiciais. Estima-se que, em 2016, as empresas americanas pagaram mais de US\$ 1,5 milhões em penalidades por não protegerem seus trabalhadores contra o ruído, e que US\$ 242 milhões são gastos anualmente na compensação de trabalhadores por PAIR. No Brasil, não há dados que permitam realizar estimativas como essas, uma vez que a subnotificação da PAIR é considerável. Mas, certamente, a PAIR é um problema, na área da saúde do trabalhador, que não está controlado.

Além dos efeitos nocivos do ruído intenso sobre a audição, há prejuízo também aos sistemas circulatório, endócrino, digestivo e neurológico, além da contribuição para o aumento do número de acidentes de trabalho; o processo de comunicação é afetado como um todo quando há a PAIR, o que também leva à deterioração da qualidade de vida do trabalhador ${ }^{3}$.

Devido a essas consequências negativas na vida do trabalhador, torna-se essencial o desenvolvimento de programas que consigam promover o conhecimento das questões relacionadas à exposição ao ruído intenso no trabalho, assim como buscar soluções para diminuir ou eliminar essa exposição, visando à preservação da audição e à promoção da saúde no trabalho ${ }^{4}$.

Em relação a essas questões, está prevista na legislação trabalhista brasileira (Consolidação das Leis Trabalhistas), através da Norma Regulamentadora NR 7, Anexo I, e da NR 9, a inclusão de trabalhadores expostos ao ruído intenso em programas de conservação auditiva (PCA) ${ }^{5,6}$, também chamados de programas de preservação auditiva.

Autores sugerem que esses programas sejam estruturados a partir de três eixos principais, constituídos por ações sobre o ambiente de trabalho, monitoramento da saúde (geral e auditiva) e ações educativas aos trabalhadores e responsáveis pela empresa, e observam que o PCA será eficiente quando contribuir para a melhoria das condições de trabalho e da saúde dos trabalhadores ${ }^{4,7}$.

Em relação ao desenvolvimento de atividades educativas como parte dos programas, a literatura relata que estas devem oferecer ao trabalhador a oportunidade de refletir mais amplamente sobre as suas condições de trabalho, sua saúde e sua qualidade de vida, além de possibilitar a compreensão sobre como o trabalho pode impactá-las negativamente, favorecendo, então, a busca de soluções ${ }^{8,9}$.

Porém, a possibilidade de o trabalhador realizar essas reflexões depende da abordagem e dos objetivos adotados pelos profissionais da saúde envolvidos na execução do programa educativo. Autores consideram que apesar dos avanços conceituais sobre saúde, promoção da saúde e prevenção de doenças, ainda há uma ação simplificada e baseada em modelos tradicionais de educação ${ }^{10,11}$, o que não favoreceria uma participação efetiva do trabalhador nesse processo.

Essas diferenças conceituais entre promoção, prevenção e educação em saúde são pontuadas por alguns autores, como Czeresnia ${ }^{12}$, que, em relação à diferença entre prevenção e promoção, afirma que prevenir é "preparar, chegar antes de, dispor de maneira que evite (dano, mal), impedir que se realize", e promover (saúde) é "dar impulso a, fomentar, originar, gerar". Para Buss ${ }^{10}$, a promoção da saúde tem exercido uma crescente influência na organização dos sistemas de saúde, mas também se apresenta contraditória com relação às suas premissas e estratégias. Segundo o autor, essas contradições são resultantes das diversas concepções de promoção da saúde adotadas por diferentes autores ou grupos, e que podem ser reunidas em duas grandes tendências: a primeira, centrada em ações sobre o comportamento dos indivíduos e mudanças de estilo de vida; e a segunda, com um enfoque mais amplo, buscando articular o tema da saúde com o das condições e qualidade de vida. A primeira tendência, segundo o autor, teria um enfoque marcadamente comportamental. Suas características incluem ações em saúde para a mudança de hábitos e estilos de vida, levando em consideração ambiente familiar e contexto cultural dos envolvidos. Dessa maneira, a promoção da saúde enfatizaria aspectos educativos vinculados aos fatores de risco comportamentais, o que traz a ideia de que poderiam ser controlados pelos sujeitos. Diferentemente, na segunda tendência, a ênfase está no papel protagonista dos determinantes gerais sobre as condições de saúde. As características dos sujeitos, as condições do ambiente nos aspectos físicos, sociais, econômicos e culturais são consideradas. 
Entre os autores que adotam a primeira concepção de promoção da saúde descrita por Buss ${ }^{10}$, a ação preventiva objetiva a persuasão dos indivíduos para a adoção de comportamentos mais saudáveis e, a partir das informações sobre os riscos para a saúde, o indivíduo é considerado o maior responsável por sua saúde ou culpado pela doença ${ }^{13}$. Em relação à prevenção auditiva no trabalho, pode-se dar como exemplos os programas educativos que centralizam suas ações na utilização de protetores auriculares como única alternativa para a preservação auditiva. Autores que adotam essa abordagem descrevem, então, diversos modelos de comportamentos em saúde, que identificam aspectos considerados fundamentais na influência de hábitos, e propõem ações para melhorar a eficácia dos treinamentos para utilização dos protetores auriculares pelos trabalhadores ${ }^{14,15}$.

E, em relação à segunda concepção sobre promoção da saúde elencada por Buss ${ }^{10}$, Lacaz ${ }^{16}$ discorre sobre as ações educativas com trabalhadores, cuja intencionalidade seria envolver os diferentes sujeitos inseridos no contexto de produção do processo saúde e doença, buscando-se ampliar a capacidade dos coletivos de compreender e interferir na sua realidade. Para tanto, o trabalhador deve ser entendido como agente de mudanças, possuidor de saberes e vivências sobre seu trabalho, que são compartilhados de forma coletiva, e que podem auxiliar na transformação de seu ambiente de trabalho.

Assim, a educação em saúde, enquanto uma prática educativa, redimensiona o papel dos profissionais como educadores, pois deve propiciar aos trabalhadores tornarem-se agentes na promoção e proteção de sua saúde, através de um processo educativo efetivado de maneira relacional e não hierárquica. Nesse contexto, o elemento central de todas as ações educativas são as pessoas (primazia das pessoas), que devem ser capazes de identificar os fatores que afetam sua saúde e, com isso, exercer maior controle sobre suas vidas. Desenvolve-se, então, a consciência nas pessoas em relação aos seus problemas de saúde, estimulando a busca de soluções e a organização para a ação coletiva ${ }^{11,13,17}$.

Então, a educação em saúde deve ser vista como estimuladora de transformações na relação saúde e trabalho. Para tanto, deve ser uma prática pedagógica planejada para que os trabalhadores desenvolvam suas capacidades de analisar, questionar, relacionar e criar, participando ativamente de seu ambiente de trabalho ${ }^{18}$.

Apesar do potencial para mudanças das ações educativas voltadas aos trabalhadores, será que suas práticas vêm sendo plenamente desenvolvidas como parte dos programas de preservação auditiva?

Nesse contexto, este artigo objetivou analisar a produção científica sobre intervenções educativas desenvolvidas para a prevenção auditiva de trabalhadores expostos ao ruído.

\section{Métodos}

Para alcançar o objetivo proposto, optou-se pelo método da revisão integrativa descritiva e analítica, tendo em vista que esse método possibilita sumarizar as pesquisas já concluídas e obter conclusões a partir de um tema de interesse. A revisão integrativa exige os mesmos padrões de rigor, clareza e replicação utilizados nos estudos primários ${ }^{19}$.

Nesta revisão, utilizou-se as seguintes etapas: seleção das questões temáticas, estabelecimento dos critérios para a seleção de estudos, definição das informações a serem extraídas dos estudos selecionados, avaliação dos estudos incluídos, interpretação dos resultados e apresentação da revisão/sínteses do conhecimento, com a análise dos principais resultados encontrados nos artigos incluídos.

A busca foi realizada nas bases de dados LILACS (Literatura Latino-Americana e do Caribe em Ciência da Saúde), MEDLINE (Medical Literature Analysis and Retrieval System Online), PubMed, SciELO e Google Scholar.

Para a busca, utilizou-se uma combinação de descritores associados pelo operador booleano "AND". No idioma português utilizou-se: promoção da saúde, audição, trabalhadores, perda auditiva provocada por ruído, educação, saúde do trabalhador; e na língua inglesa: "health promotion", "hearing", "workers", "noise-induced hearing loss", "education", "occupational health". Foram também utilizadas as referências bibliográficas dos artigos selecionados para a localização de outros artigos.

Os artigos selecionados atenderam aos seguintes critérios de inclusão: (1) ser disponibilizado na íntegra; (2) publicado no período de 2005 a 2016; (3) conter proposta de intervenção junto aos trabalhadores sob risco de perda auditiva e serem estudos sobre programas de saúde no trabalho, com foco na saúde auditiva. Os critérios de exclusão foram: (1) programas de saúde sem foco na população trabalhadora; (2) ações não envolvendo saúde auditiva; (3) outro idioma diferente de português e inglês, (4) estudo publicado fora do limite temporal definido.

Após o levantamento preliminar dos artigos, realizou-se a leitura dos títulos e resumos para identificar a pertinência com o objeto estudado e, posteriormente, a seleção dos artigos e leitura na íntegra. A partir da composição de um banco de dados com os artigos que atenderam aos critérios, foi realizado um fichamento para sistematização das informações, 
organizadas nas dimensões: título da pesquisa, autores, ano de publicação, revista de publicação e base de dados de indexação.

A análise do conteúdo dos artigos selecionados considerou: ano de publicação, autores, local do estudo, objetivos, população do estudo (gênero, atividade econômica, idade, tempo de serviço e escolaridade), abordagem, estratégias operacionais na realização da ação educativa, principais resultados e conclusões.

Após a sistematização dos dados, foi realizada uma análise crítica das abordagens utilizadas nas ações educativas e seus principais resultados.

\section{Resultados}

Inicialmente, foram identificados 137 artigos (77 internacionais e 60 nacionais). Considerando-se os critérios de inclusão e exclusão, a verificação da coerência com a temática pesquisada e a eliminação de artigos repetidos em mais de uma base de dados, foram efetivamente incluídos no estudo 16 artigos: 9 internacionais e 7 nacionais.

As características gerais dos artigos selecionados encontram-se no Quadro 1 (internacionais) e Quadro 2 (nacionais). As informações sistematizadas incluíram: autoria e localização geográfica; descrição da população estudada (atividade de trabalho, número de sujeitos, gênero, média ou faixa de idade em anos, média ou faixa de tempo de serviço em anos, grau de escolaridade); abordagem e estratégias operacionais das intervenções educativas; principais resultados. Alguns artigos não continham todas as informações.

As categorias profissionais incluídas nos estudos foram: mineiros de carvão ${ }^{20}$, operários da construção civil $^{14,21-23}$, carpinteiros ${ }^{24}$, bombeiros ${ }^{15}$, operários de empresa de equipamentos eletrônicos ${ }^{25}$, agricultores ${ }^{26}$, trabalhadores de empresa frigorífica ${ }^{27,31}$, teleoperado$\mathrm{res}^{28}$, trabalhadores de universidade ${ }^{29,33}$, pescadores industriais ${ }^{30} \mathrm{e}$ trabalhadores de empresa alimentícia ${ }^{32}$.

As amostras dos estudos variaram em tamanho, de 11 a 491 trabalhadores. A maioria dos trabalhadores era do gênero masculino, com idades variando entre 16 e 50 anos, tempo de serviço na função de 2,6 a 25 anos e escolaridade predominando no ensino médio completo ou incompleto, que equivale de 9 a 11 anos de escolaridade (nos artigos nacionais), e high school, que equivale de 9 a 12 anos de escolaridade (nos artigos internacionais).

Os participantes de todos os estudos foram descritos como expostos ao ruído intenso ocupacional. Todavia, essas descrições foram frequentemente baseadas em métodos de medição de níveis de pressão sonora que não foram claramente descritos.
Entre os nove estudos internacionais, oito $^{4,15,20-24,26}$ foram realizados nos Estados Unidos e tinham objetivos similares para as intervenções educativas: pretendiam aumentar a intenção de uso e a utilização de protetores auriculares como prática de prevenção de perdas auditivas no trabalho. Já no estudo coreano ${ }^{25}$, o objetivo foi a redução do nível de ruído no ambiente de trabalho e a diminuição da exposição, para controle da PAIR.

Entre os sete estudos nacionais, três $28,29,33$ foram desenvolvidos em São Paulo, dois ${ }^{31,32}$ no Paraná (Curitiba) e dois ${ }^{27,30}$ em Santa Catarina (não mencionavam as cidades), os objetivos versaram sobre: (1) ação conjunta com trabalhadores para capacitá-los como promotores de sua saúde, enquanto atores sociais; (2) avaliação do conhecimento sobre proteção auditiva; (3) treinamento com ênfase na importância da proteção auditiva; (4) caracterização de participantes de ação voltada à preservação auditiva; (5) avaliação da percepção de conforto e utilização de protetor auricular após intervenção; (6) avaliação de oficinas educativas para trabalhadores expostos ao ruído; (7) eficácia na colocação de protetores auriculares em grupos com e sem treinamentos.

Ao analisarem-se os delineamentos das pesquisas realizadas, verificou-se que 15 estudos utilizaram a abordagem quantitativa, e um estudo nacional ${ }^{28}$ desenvolveu a pesquisa com métodos qualitativos.

A descrição da abordagem utilizada nas intervenções educativas realizadas nos oito estudos desenvolvidos nos Estados Unidos ${ }^{14,15,20-24,26}$ foram baseadas na Teoria Comportamental, que serviu de base para o Modelo de Crenças na Saúde (Health Belief Model) e o Modelo de Promoção da Saúde (Health Promotion Model), ambos referenciados pelos estudos. O foco desses estudos foi nas mudanças de crenças e atitudes dos trabalhadores para maior adesão ao uso de protetores auriculares. $\mathrm{O}$ artigo coreano ${ }^{25}$ também se baseou em abordagens comportamentais para desenvolver uma cultura de segurança, porém, com enfoque também em mudanças administrativas e nos sistemas de gerenciamento relacionados à segurança no trabalho.

Dos sete estudos nacionais, três ${ }^{27,29,33}$ utilizaram abordagens comportamentais, porém sem referência à base teórica ou autores, e visavam a utilização de protetores auriculares; quatro ${ }^{28,30-32}$ utilizaram ações educativas, sendo que desses, dois ${ }^{30,31}$ com estratégias de único encontro e sem mencionar o referencial teórico utilizado, mas também com foco na utilização de protetores auriculares. Os outros dois com ações educativas ${ }^{28,32}$ utilizaram mais de um encontro com os trabalhadores e visaram capacitá-los como atores sociais do processo de saúde. Dentre esses, um estudo $^{32}$ referiu utilizar a metodologia participativa nas oficinas educativas desenvolvidas. 


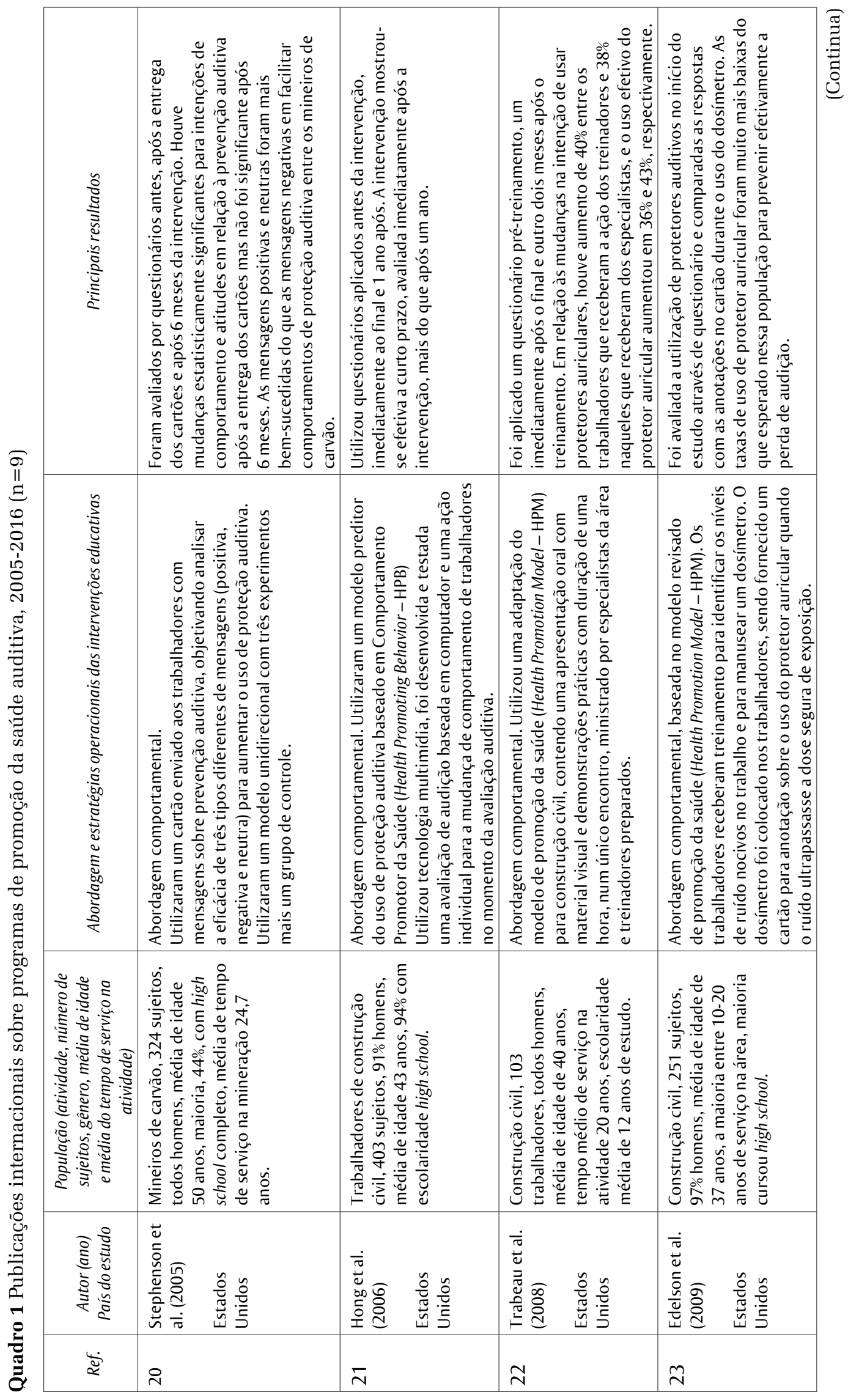




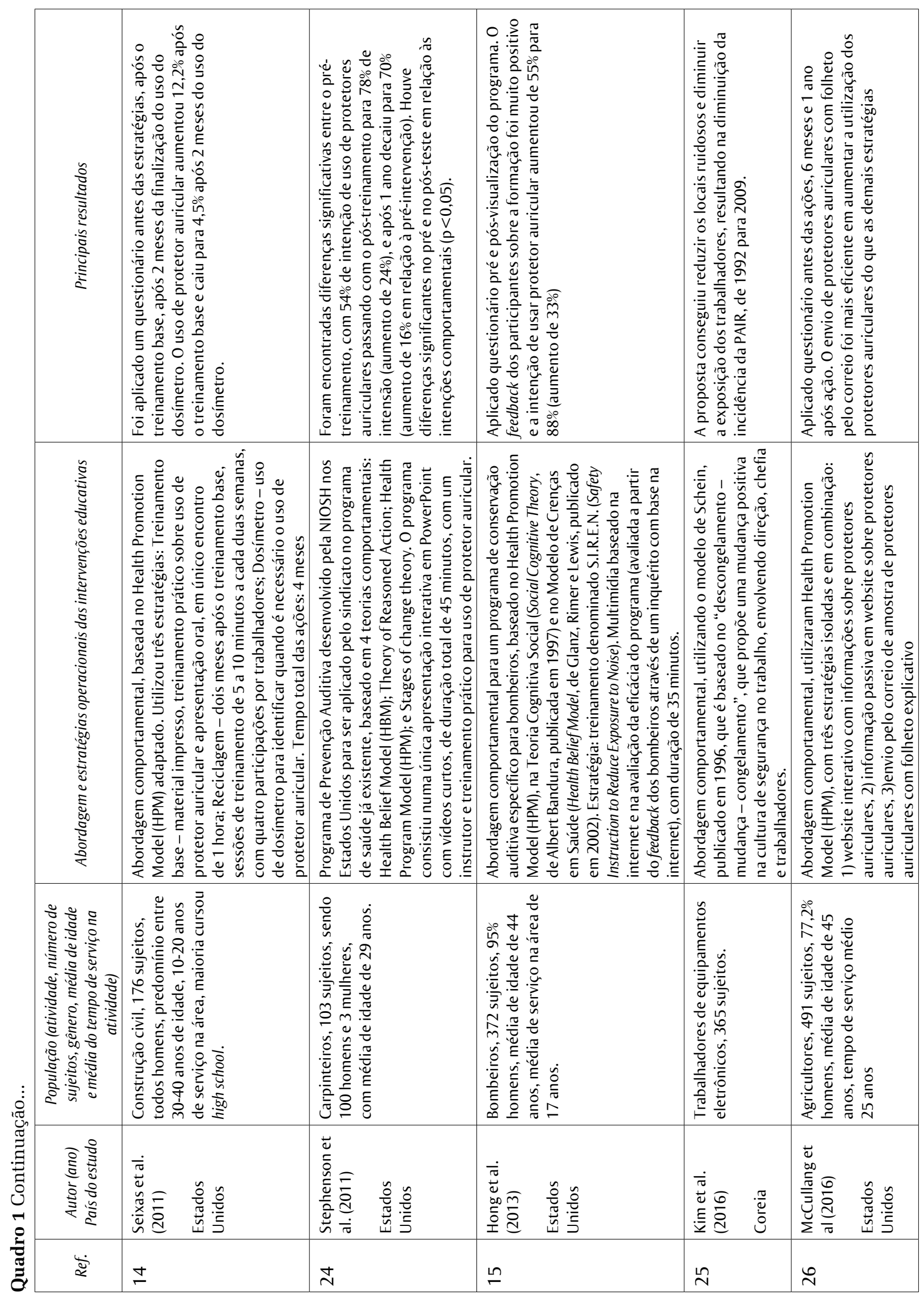




\begin{tabular}{|c|c|c|c|c|c|c|c|}
\hline 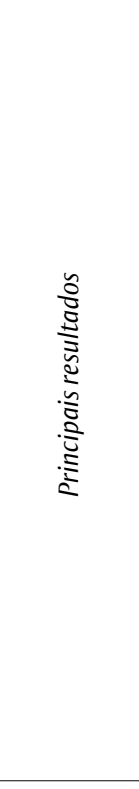 & 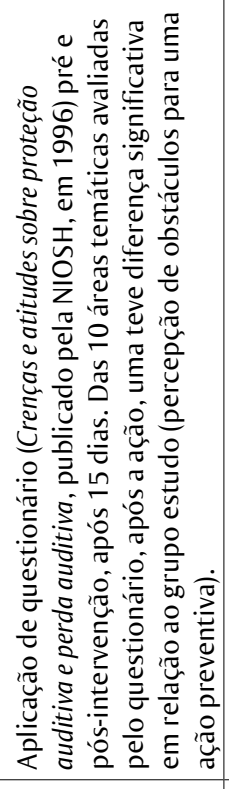 & 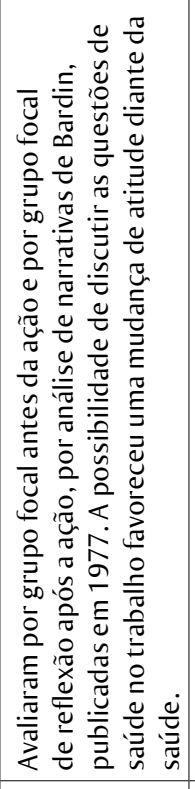 & 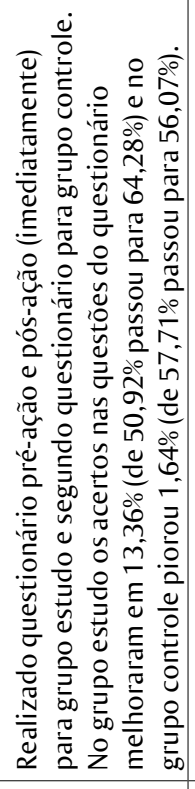 & 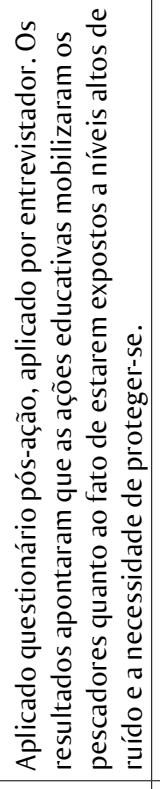 & 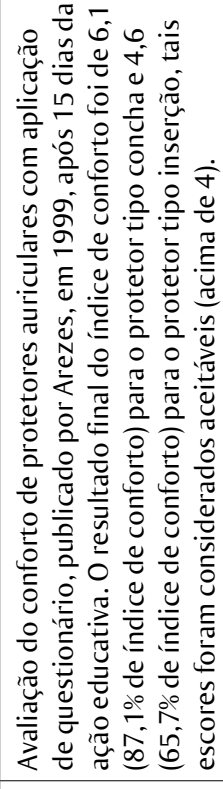 & 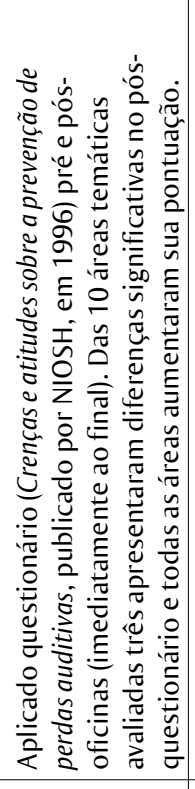 & 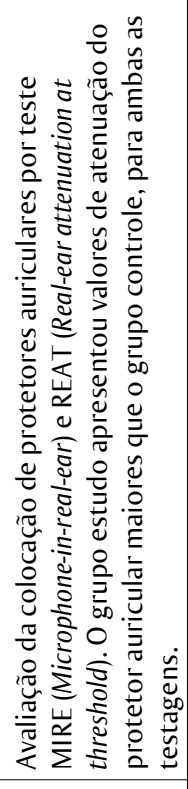 \\
\hline 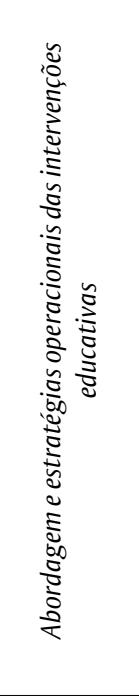 & 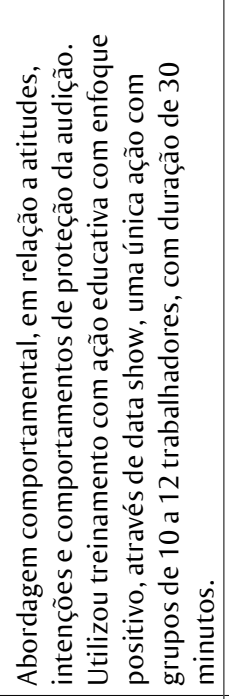 & 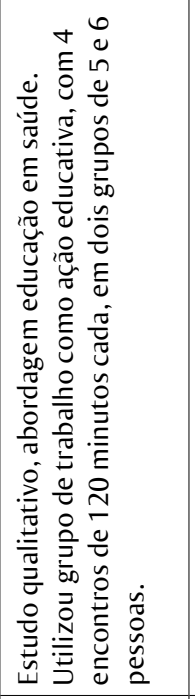 & 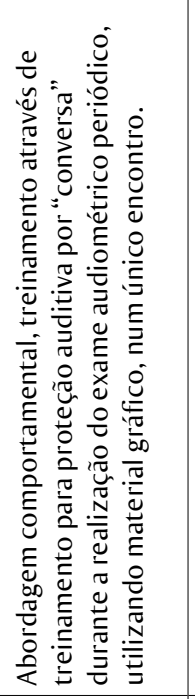 & 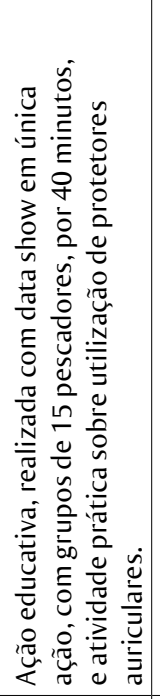 & 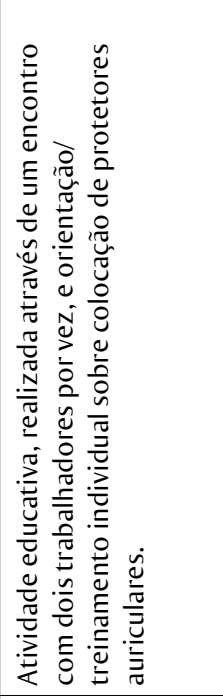 & 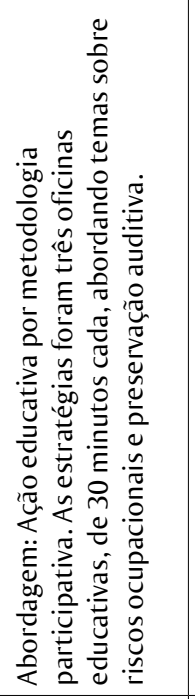 & 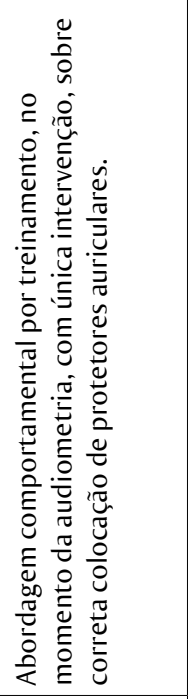 \\
\hline 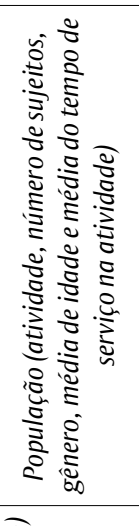 & 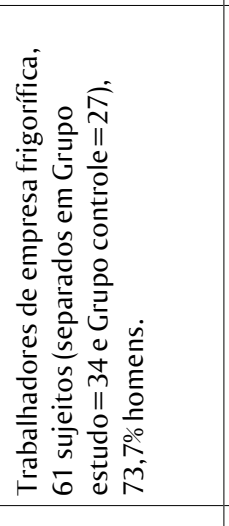 & 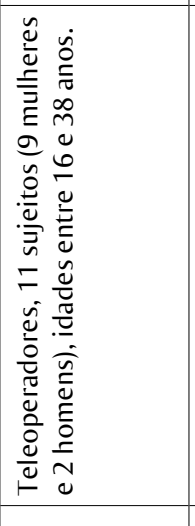 & 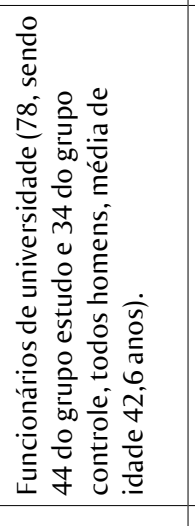 & 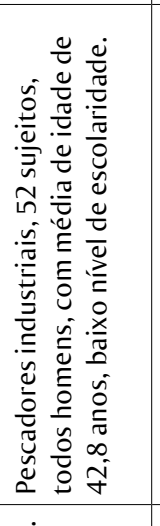 & 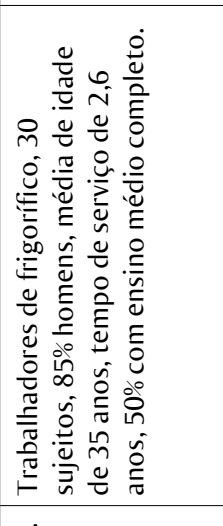 & 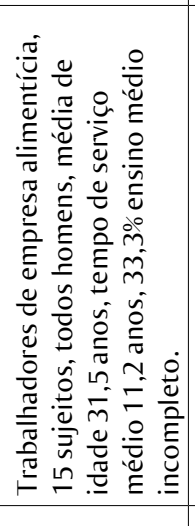 & 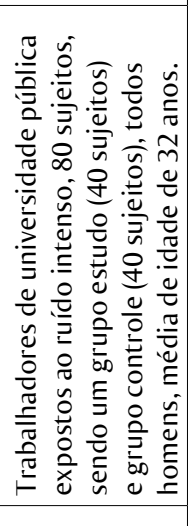 \\
\hline 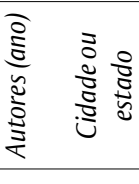 & 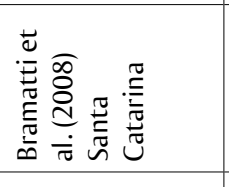 & 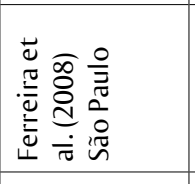 & 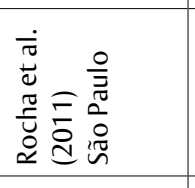 & 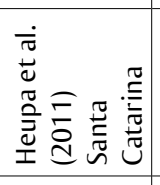 & 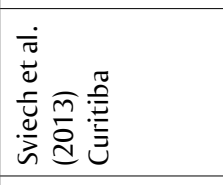 & 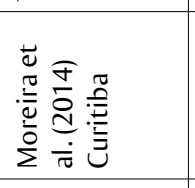 & 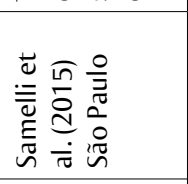 \\
\hline$\ddot{\check{\varpi}}$ & $\grave{N}$ & $\stackrel{\infty}{\sim}$ & নิ & m & $\bar{m}$ & $\tilde{n}$ & $\tilde{m}$ \\
\hline
\end{tabular}


Em relação às estratégias utilizadas entre os nove estudos internacionais, sete $\mathrm{e}^{14,15,20-22,24,26}$ mencionaram a realização de treinamentos em um único momento, sendo que um deles realizou uma ação e uma reciclagem ${ }^{14}$ e outro estava sem informação ${ }^{23}$. As estratégias empregadas foram diversificadas, envolvendo treinamentos por: material impresso, flip-chart, material multimídia, website, palestra e treinamento prático.

Nos estudos nacionais, cinco ${ }^{27,29-31,33}$ utilizaram apenas uma intervenção e dois ${ }^{28,32}$ realizaram mais de dois encontros sequenciais. As estratégias utilizaram: material impresso, material multimídia, palestra, treinamento prático, avaliação de colocação de protetores auriculares por testagens, oficinas educativas e grupos de trabalho.

Entre os artigos internacionais que avaliaram os resultados da intervenção para promover proteção auditiva e aumentar o uso de protetores auditivos, oito $^{14,15,20-24,26}$ utilizaram questionários pré e pós-aÇão, sendo que quatro ${ }^{14,15,22,23}$ avaliaram os resultados em prazos imediatos à intervenção ou até em um período de 2 meses, um $^{20}$ avaliou imediatamente e após 6 meses da ação, um ${ }^{26}$ avaliou imediatamente, após 6 e 12 meses da ação, dois ${ }^{21,24}$ avaliaram imediatamente e após 12 meses da ação. O artigo que analisou as mudanças em relação ao controle do ruído avaliou os resultados a partir da análise de audiometrias realizadas num período de 17 anos $^{25}$.

Entre os sete artigos nacionais, três ${ }^{27,29,32}$ estudos aplicaram questionários pré e pós-ação (um deles foi após 15 dias do término da ação e em dois deles foi imediatamente após a ação); dois estudos somente aplicaram um questionário pós-ação (um deles imediatamente após a ação ${ }^{30}$ e outro após 15 dias de seu término ${ }^{31}$ ); um $^{28}$ estudo utilizou o grupo focal pré-ação educativa e o grupo focal de reflexão após a ação; e um ${ }^{33}$ estudo utilizou testes de colocação de protetores auriculares após o treinamento.

Entre os estudos internacionais, os principais resultados sobre as intervenções realizadas referiram melhoria na intenção de uso e na utilização de protetores auriculares entre os trabalhadores analisados quando a avaliação ocorreu imediatamente após a ação, variando esse aumento de 12,2\% a $40 \%$ (dependendo do estudo) de trabalhadores com a intenção de utilização de protetores auriculares. Porém, os estudos que fizeram as avaliações pós-intervenção após um período de tempo maior, observou-se um decréscimo nesses percentuais de trabalhadores com intenção de utilização de protetores auriculares em quase $50 \%$. Um estudo ${ }^{23}$ não observou melhorias significativas após a ação. E, $u^{25}$ estudo comprovou a prevenção da perda auditiva através da redução dos casos de PAIR.
Em relação aos resultados nos estudos nacionais, dois estudos que utilizaram o mesmo questionário (questionário Crenças e atitudes sobre proteção auditiva e perda auditiva), que avalia 10 áreas temáticas (a saber: percepção de suscetibilidade de adquirir uma perda auditiva; percepção da severidade das consequências de perda auditiva; percepção de benefícios de uma ação preventiva; percepção de obstáculos para ação preventiva/conforto; percepção de obstáculos para ação preventiva/atenuação de sons importantes; percepção de obstáculos para ação preventiva/comunicação; percepção de obstáculos para ação preventiva/conveniência e disponibilidade de proteção auditiva; intenções de comportamento; normas sociais; e autoeficácia), observaram diferenças significativas nos resultados pré e pós-aplicação em apenas uma área temática (percepção de obstáculos para ação preventiva/conforto) num estudo ${ }^{27}$ e em três áreas (percepção de suscetibilidade de adquirir uma perda auditiva; percepção de benefícios de uma ação preventiva; intenções de comportamento) no outro estudo ${ }^{32}$; outro estudo observou uma melhora de $13,36 \%$ nos acertos de questões pelo grupo intervenção ${ }^{29}$; no estudo que fez avaliação do conforto do protetor auricular, os índices conseguidos após a intervenção $(87,1 \%$ de índice de conforto para o protetor tipo concha e $65,7 \%$ de índice de conforto para o protetor tipo inserção) ${ }^{31}$ foram considerados satisfatórios; e o estudo que utilizou o grupo focal ${ }^{28}$ observou, pela análise das narrativas, mudanças nas atitudes preventivas dos participantes.

\section{Discussão}

Na realização desta revisão da literatura, foram observadas publicações científicas sobre intervenções educativas voltadas à prevenção auditiva de trabalhadores em maior número na produção internacional do que na nacional, predominando aquelas desenvolvidas nos Estados Unidos. Isso talvez porque as primeiras referências sobre ações preventivas para as PAIR surgiram na legislação dos Estados Unidos, em 1970. Nesse período, foi emitida uma regulamentação sobre exposição ao ruído ocupacional que mudou os requisitos normativos e de fiscalização daquele país. Em 1990, foram lançadas as diretrizes para os programas de preservação auditiva e instituída a sua obrigatoriedade em todas as indústrias que apresentassem níveis de ruído elevados. As determinações americanas influenciaram as legislações de outros países, como o Brasil, com as alterações das NR 7 e NR $9^{4}$.

Em relação à população dos estudos, observou-se que os trabalhadores do setor secundário predominaram nos estudos internacionais e nacionais. Porém, 
há também estudos com trabalhadores do setor primário (agricultores e pescadores) e terciário (bombeiros, teleoperadores e profissionais de universidade). A ocorrência de estudos principalmente no setor secundário pode ser devido à uma maior concentração de casos de PAIR nesse setor, pelos riscos das atividades ali concentradas e um maior contingente de trabalhadores expostos ${ }^{34,35}$.

A maioria dos trabalhadores dos estudos selecionados pertencia ao gênero masculino, apresentava faixa etária e tempo de serviço diversificados, mas grau de escolaridade similar, entre 9 e 12 anos de ensino, em média. A literatura relata predomínio de PAIR no gênero masculino justificando que trabalhos perigosos são preferencialmente executados por homens ${ }^{34,35}$.

Em relação às intervenções educativas para a prevenção auditiva de trabalhadores expostos ao ruído, todos os estudos internacionais desenvolvidos nos Estados Unidos realizaram intervenções para modificar comportamentos, identificando atitudes, crenças e intenções em relação à utilização de protetores auriculares, buscando favorecer o aumento de sua utilização nos locais de trabalho. Já o estudo coreano, apresentou uma visão ampliada da prevenção auditiva, focando nas mudanças administrativas e no ambiente de trabalho, tornando-o menos ruidoso. Dentre os estudos nacionais, dois também apresentaram essa visão mais ampliada, mas os demais, cinco, enfatizaram a utilização de protetores auriculares. A literatura afirma que os programas de preservação auditiva devem ir para além do uso de protetores auditivos, porém, ainda é comum que sejam desenvolvidos com esse foco como principal medida de prevenção da PAIR ${ }^{36,37}$. Uma das possíveis explicações para esse fato é o desconhecimento das medidas de controle de ruído. Num estudo com supervisores de produção de uma empresa automobilística de grande porte, os autores observaram um conhecimento superficial desses profissionais sobre a prevenção auditiva, e que consideravam a utilização de protetores auriculares como a única forma de prevenir a PAIR ${ }^{38}$. Outra explicação possível para a adesão exclusiva dos PCA à utilização de protetores auriculares seria o fato de que os investimentos em medidas coletivas de controle de ruído apresentam custo mais elevado em comparação com os protetores auriculares ${ }^{37}$. A legislação brasileira prioriza o controle do ruído por medidas coletivas, porém, permite a utilização de protetores auriculares até que tais medidas sejam implementadas, o que pode favorecer unicamente a adoção dos protetores auriculares por anos ${ }^{6}$.

Em relação à base teórica apresentada nos estudos analisados nesta revisão, a maioria, quinze, utilizou uma abordagem comportamental para o desenvolvimento das intervenções educativas. Esse foco das intervenções educativas em estratégias que auxiliem na motivação e no suporte de comportamentos desejáveis do trabalhador está relacionado à primeira concepção sobre promoção da saúde ${ }^{10}$.

Nessa direção, nos Estados Unidos, autores como Pender et al. ${ }^{39}$ elaboraram um modelo teórico sobre promoção da saúde (HPM - Health Promotion Model) para ser utilizado com trabalhadores expostos a ruído intenso. A proposta foi baseada no Health Belief Model (HBM), descrita por Rosenstock ${ }^{40}$ e por outros autores na década de 1970, que sugere que as respostas comportamentais a um risco para a saúde, como a exposição ao ruído intenso que gera a PAIR, (grifo dos autores) estão relacionadas a vários fatores mensuráveis, como: suscetibilidade a um risco para saúde, crença na gravidade do problema de saúde, crenças na eficácia das medidas de proteção propostas, crenças em relação às barreiras à adoção de comportamentos protetores e na própria capacidade de superar essas barreiras. Esses modelos resultam em estratégias de treinamentos baseadas em abordagens de mudanças de comportamento, ao identificar fatores que parecem estar associados às atitudes, crenças e intenções comportamentais dos trabalhadores.

Na proposta do $\mathrm{HPM}^{39}$, pode-se observar a responsabilização do trabalhador, pois as ideias sobre saúde e capacidade para o trabalho e suas relações com a promoção da saúde no trabalho, podem ser resumidas como: (1) a saúde e a capacidade para o trabalho são produtos da responsabilidade individual e dos comportamentos relacionados à saúde; (2) a saúde é um produto de diversos fatores, muitos dos quais estão além do controle do indivíduo. Trata-se, então, de um modelo para explicar e predizer os estilos de vida favoráveis à saúde, que incorporam conceitos cognitivos-perceptuais, tais como: a percepção da autoeficácia e a percepção de benefícios e barreiras, tanto individuais como organizacionais, para implementar-se um comportamento. Pesquisadores utilizaram essa proposta adaptando-a para a percepção dos riscos da exposição ocupacional ao ruído intenso e o reforço na utilização de protetores auriculares ${ }^{14,41}$. Segundo os autores, esse modelo consegue aumentar a porcentagem de trabalhadores que utilizam protetores auriculares em até $50 \%^{39}$. A proposta do HPM, do ponto de vista da concepção de um programa de treinamento para prevenção de perda auditiva, complementaria os modelos HBM e Theory of Reasoned Action, pois adicionaria ênfase em modelos positivos que encorajam mudanças comportamentais e o valor de solicitar-se um compromisso público do trabalhador para o comportamento promovido ${ }^{24}$.

Já nos estudos nacionais, não há claramente referências às bases teóricas utilizadas. Porém, quatro estudos nacionais realizaram o que nomearam de ações educativas, no entanto, somente em dois $^{28,32}$ deles é possível identificar propostas que se 
relacionam com a compreensão da promoção da saúde com enfoque mais amplo, buscando articular a saúde com a temática das condições e qualidade de vida ${ }^{10}$ : um deles ${ }^{28}$ buscou capacitar os trabalhadores como atores sociais do seu processo de saúde e o outro ${ }^{32}$ utilizou a metodologia participativa nas oficinas educativas desenvolvidas. Segundo Vasconcellos ${ }^{42}$, a prática educativa pautada na metodologia participativa é considerada como um processo de instrumentalização dos indivíduos e de grupos sociais para que identifiquem e analisem os problemas de saúde com o intuito de elaborar propostas de solução. Porém, observou-se que ainda não está plenamente incorporada na maioria das ações voltadas aos trabalhadores, talvez por falta de formação de muitos profissionais da saúde e segurança nessa abordagem ${ }^{13,17,43}$

Quanto às estratégias utilizadas para as intervenções educativas desenvolvidas com os trabalhadores, observou-se que estavam de acordo com as abordagens selecionadas. Assim, em grande parte dos artigos analisados, optou-se por palestras ${ }^{27,30}$, apresentações únicas de estratégias e treinamentos; já os encontros sequenciais, como oficinas e as discussões por meio de grupo focal de reflexão ${ }^{28,32}$, foram adequadas às propostas que se identificavam como sendo de ações educativas ${ }^{7,13,43}$.

Observou-se que os estudos utilizaram e aplicaram questionários pré e pós-intervenção educativa para verificar a efetividade das ações realizadas. Instrumentos como questionários são ferramentas estáveis e viáveis para a verificação da efetividade de programas educativos, sendo que os mesmos prestam-se para a avaliação do nível de conhecimento de trabalhadores submetidos a intervenções, assim como para a estimativa do alcance e da efetividade da intervenção realizada ${ }^{27}$. Porém, os resultados dos estudos foram bem diversificados, inclusive em função do tempo esperado para a aplicação do segundo questionário (pós-intervenção). Nenhum estudo conseguiu um percentual igual ou maior que $50 \%$ de melhora após a intervenção imediata, ficando com percentuais ainda menores quando a avaliação posterior foi realizada após alguns meses da intervenção ${ }^{39}$. No estudo que utilizou a metodologia participativa ${ }^{32}$ houve $30 \%$ de melhora, sendo este o melhor resultado dentre os estudos nacionais.

Os índices de resultados alcançados pelos estudos internacionais após intervenção, ou seja, a adesão dos trabalhadores à intenção de uso e utilização de protetores auriculares, que foi de $12,2 \%$ a $40 \%$ na avaliação imediata, porém, decresceram em 50\% após um período mais prolongado, assim como a baixa adesão às mudanças de atitudes na maioria dos estudos nacionais (principalmente entre os estudos que pretendiam aumentar a utilização de protetores auriculares), podem ser explicados por três fatores, descritos a seguir:
1) Desconsideração das experiências e dos conhecimentos prévios dos trabalhadores sobre riscos e prevenção auditiva: observou-se que em alguns estudos já havia informações sobre prevenção auditiva entre os trabalhadores, identificadas pelo questionário pré-intervenção ${ }^{15,24,27}$. Então, negligenciar o saber e a vivência do trabalhador e acreditar que seus problemas de saúde se devem à ignorância sobre as normas de saúde e segurança no trabalho, e que a mudança de atitudes e comportamentos individuais, por si só, solucionariam os problemas de saúde como um todo, pode induzir a erros ${ }^{7,18,43}$.

2) Realização de intervenções educativas baseadas na transmissão de informação objetivando, exclusivamente, a mudança de comportamento: a maioria dos estudos que pretendiam a mudança de atitudes em relação à utilização de protetores auriculares e que aderiram à abordagem comportamental pioraram sua avaliação com maior tempo decorrido após a ação de intervenção, ou seja, os novos hábitos e atitudes, quando instalados (e se instalados), tenderam a não se manter em médio e longo prazo. Isso pode ter ocorrido porque essa abordagem apresenta uma visão simplificada de promoção da saúde, na qual priorizam-se as mudanças de estilos de vida, o modelo tradicional da educação e a compreensão de que prevenção da doença é sinônimo de promoção da saúde ${ }^{10,11,13,43}$. A ênfase, no entanto, deve ser direcionada ao processo de capacitação dos indivíduos e grupos, objetivando a transformação da realidade em que se encontram, ampliando a sua percepção das causas políticas e sociais envolvidas, tornando-se assim efetiva as transformações. Uma prática educativa assim pautada será um processo de instrumentalização dos trabalhadores, para que analisem seus problemas de saúde com a finalidade de elaborar propostas de solução ${ }^{10,11,13,43}$. Assim sendo, a promoção da saúde passaria a ser vista como uma estratégia mediadora entre trabalhadores e seu ambiente de trabalho, visando ampliar a participação deles na modificação dos determinantes do processo saúde-doença no trabalho ${ }^{7,10,44}$. Porém, essa maneira de atuar exige dos profissionais uma outra postura, tornando-se educadores capazes de auxiliar os trabalhadores a serem ativos, agentes na promoção e proteção de sua saúde. Para tanto, os educadores devem propiciar um processo de aprendizagem de uma maneira relacional e não hierárquica, promovendo a autonomia dos trabalhadores, para que, em conjunto, possam compreender a saúde como resultante 
das condições de vida e trabalho e conquistar o direito à vida com ética e dignidade ${ }^{11,13,44}$.

3) Desconsideração das mudanças nos ambientes e na organização do trabalho: a maioria dos estudos não contemplou a discussão sobre mudanças no ambiente de trabalho para redução da exposição ao ruído, colocando sobre o trabalhador toda a responsabilidade sobre o sucesso ou fracasso da garantia de sua audição, desconsiderando as limitações do uso exclusivo de protetor auricular em si. Um dos princípios básicos de saúde e segurança no trabalho é a associação de medidas ativas (uso de equipamentos de proteção individual, como os protetores auriculares) que são subordinadas ao comportamento do trabalhador, com as ditas medidas passivas, aquelas que agem independentemente do trabalhador ${ }^{45}$. A situação ideal para a prevenção auditiva é o controle do ruído no ambiente de trabalho, seja por medidas administrativas ou por medidas tecnológicas. A utilização de protetores auriculares como medida exclusiva de proteção vem sendo apontada na literatura como não suficiente para garantir a audição dos trabalhadores. Diversos fatores dificultam a utilização eficiente de protetores auriculares, a começar pelos níveis de ruído muito intensos que não são possíveis de serem atenuados unicamente pelos protetores auriculares ${ }^{46}$. Além disso, fatores como as técnicas de colocação dos protetores auriculares, o conforto, a aceitação do usuário, o custo e a durabilidade desses equipamentos, problemas de comunicação, segurança e higiene, entre outros, interferem na utilização eficaz dos protetores auriculares ${ }^{3,47}$.

Assim, os resultados dos estudos analisados na presente revisão indicam a insuficiência da adoção dos protetores auriculares como medida exclusiva de proteção auditiva.

A partir dos aspectos elencados nesta revisão de literatura, percebeu-se a necessidade de uma compreensão ampliada sobre os programas de conservação auditiva por parte dos profissionais da saúde e segurança do trabalho, a fim de desenvolverem ações para além do treinamento da utilização adequada de protetores auriculares ${ }^{7}$. Na intenção de ampliar-se esse olhar, é necessário também incluir ações de prevenção dirigidas a outros riscos para a audição, que ainda não são considerados, como os agentes químicos ototóxicos, ou o sinergismo do ruído com outros agentes de riscos (altas temperaturas, esforço físico excessivo, vibrações, entre outros), além de considerar a interação dos agentes de risco, como um todo, como cargas de trabalho.
Igualmente importante é a realização de ações educativas que superem uma abordagem comportamental e que contribuam com a promoção da saúde e prevenção, favorecendo a participação do trabalhador como agente de mudanças, possuidor de saberes e vivências sobre seu trabalho e capaz de intervir para transformar o seu ambiente de trabalho ${ }^{4,7,16,43}$.

Nesse sentido, a prática educativa deve ser inserida numa metodologia de participação, que, para além de métodos de persuasão ou de transferência de informação, seja um processo de capacitação dos indivíduos e grupos para a transformação da realidade em que vivem ${ }^{13,41}$.

O diálogo, então, constitui o cerne dessa proposta educativa, em que educador e educando são sujeitos de um processo, em uma relação horizontal, na qual crescem juntos, porquanto os indivíduos se educam entre si, mediatizados pelo mundo ${ }^{48}$.

\section{Conclusão}

Nos artigos analisados, pode-se observar que a maioria dos estudos utiliza as intervenções educativas para melhorar a intenção de uso e a utilização de protetores auriculares, porém, sem atingir patamares superiores a $50 \%$ de adesão dos trabalhadores após seis ou doze meses da ação.

Os resultados das publicações científicas podem ser considerados como parâmetros importantes para a realização de intervenções sobre os riscos e de ações educativas para trabalhadores expostos a ruído, indicando a necessidade de olhar mais ampliado, não restrito aos protetores auriculares.

Para tanto, a visão dos profissionais de saúde e segurança do trabalho precisa de novo enfoque, através de uma atuação que propicie a compreensão sobre promoção da saúde e a prevenção numa perspectiva que considere as relações entre as condições de trabalho e a qualidade de vida no trabalho.

Assim, as ações educativas voltadas à prevenção devem ter como principal objetivo a conscientização dos trabalhadores e responsáveis pela empresa sobre as consequências para a saúde ocasionadas pelos agentes agressivos existentes nos ambientes laborais, por meio de ações educativas mais abrangentes e participativas, associadas a medidas de controle ambiental, visando à busca de soluções efetivas.

A partir desta revisão, sugere-se uma melhor capacitação dos profissionais de saúde do trabalhador para melhor compreensão sobre as ações de prevenção e de promoção da saúde nos locais de trabalho de modo a buscar a superação dos problemas relacionados à qualidade de vida dos trabalhadores. 


\section{Contribuições de autoria}

Os autores contribuíram igualmente no desenvolvimento do trabalho e na elaboração e aprovação do texto publicado.

\section{Referências}

1. Verbeek JH, Kateman E, Morata TC, Dreschler WA, Mischke C. Interventions to prevent occupational noise-induced hearing loss: a cochrane systematic reviews. Int J Audiol. 2014;53(supl 2):584-96.

2. Occupational Safety and Health Administration OSHA. Safety and health topics: noise and hearing conservation [Internet]. 2011 [cited 2018 Sep 18]. Available from: https://www.osha.gov/SLTC/ noisehearingconservation/

3. Gonçalves CGO, Lüders D, Guirado DS, Albizu EJ, Marques JM. Perception of hearing protectors by workers that participate in hearing preservation programs: a preliminary study. CoDAS. 2015;27(4):309-18.

4. Gonçalves CGO. Saúde do trabalhador: da estruturação à avaliação de programas de preservação auditiva. São Paulo: Roca; 2009.

5. Brasil. Ministério do Trabalho e Emprego. Portaria da Secretaria de Segurança e Saúde no Trabalho $\mathrm{n}^{\circ}$ 19, de 9 de abril de 1998. Diário Oficial da União. 22 abr 1998. Seção 1:64.

6. Brasil. Ministério do Trabalho e Emprego. Portaria da Secretaria de Segurança e Saúde no Trabalho $\mathrm{n}^{\circ} 25$, de 29 de dezembro de 1994. Aprova o texto da Norma Regulamentadora $\mathrm{n}^{\mathrm{o}} 9$ - Programa de Prevenção de Riscos Ambientais. Diário Oficial da União. 30 dez 1994. Seção 1:21280-2. Republicada em 15 dez 1995. Seção 1:1987-9.

7. Gonçalves CGO, Souza MT, Masson MLV. Saúde do trabalhador e fonoaudiologia: perspectivas e desafios. In: Marchezan I, Silva HJ, Tomé MC, organizadores. Tratado das especialidades em fonoaudiologia. $2^{\mathrm{a}}$ ed. São Paulo: GEN; 2014. p. 730-6.

8. Cavalcante CAA, Nóbrega JAB, Enders BC, Medeiros SM. Promoção da saúde e trabalho: um ensaio analítico. Rev Eletr Enf [Internet]. 200 8[acesso em 2017 ago 5];10(1):241-8. Disponível em: http://www.fen.ufg.br/revista/v10/n1/v10n1a23. htm

9. Cazón RL, Mafra C, Borges JM, Boger ME, Ildefonso SG, Souza V. Educação para a saúde no trabalho. Lect educ fís Deportes [Internet]. 2007 [acesso 2017 set 17];12(112):1. Disponível em: http://www. efdeportes.com/efd112/educacao-para-saude-notrabalho.htm

10. Buss PM. Uma introdução ao conceito de promoção da saúde. In: Czeresnia D, Freitas CM, organizadores. Promoção da saúde: conceitos, reflexões, tendências. Rio de Janeiro: Fiocruz; 2003. p. 15-38.
11. Heidmann ITSB, Almeida MCP, Boehs AE, Wosny AM, Monticelli M. Promoção à saúde: trajetória histórica de suas concepções. Texto \& contexto enferm. 2006;15(2):352-8.

12. Czeresnia D. O conceito de saúde e a diferença entre prevenção e promoção. In: Czeresnia D, Freitas CM, organizadores. Promoção da saúde: conceitos, reflexões, tendências. Rio de Janeiro: Fiocruz; 2003. p. 39-53.

13. Monteiro Smeke EL, Soares de Oliveira NL. Educação em saúde e concepções de sujeito. In: Vasconcelos EM. A saúde nas palavras e nos gestos: reflexões da rede de educação popular e saúde. São Paulo: HUCITEC; 2001. p. 115-36.

14. Seixas NS, Neitzel R, Stover B, Sheppard L, Daniell B, Edelson J, et al. A multi-component intervention to promote hearing protector use among construction workers. Int J Audiol. 2011;50(1):46-56.

15. Hong O, Eakin BL, Chin DL, Feld J, Vogel S. An internet-based tailored hearing protection intervention for firefighters: development process and users' feedback. Health Promot Pract. 2013;14(4):572-9.

16. Lacaz FAC. O campo saúde do trabalhador: resgatando conhecimento e práticas sobre as relações trabalho-saúde. Cad Saúde Pública. 2007;23(4):757-66.

17. Aerts D, Alves GG, La Salvia MW, Abegg C. Promoção de saúde: a convergência entre as propostas da vigilância da saúde e da escola cidadã. Cad Saúde Pública. 2004;20(4):1020-8.

18. Mucillo M. Outras cores e contornos, pintando a educação em segurança e saúde do trabalhador. In: Salin CA, Carvalho L, organizadores. Saúde e segurança no ambiente de trabalho: contexto e vertentes. São Paulo: Fundacentro; 2002. p. 127-37.

19. Beyea SC, Nicoll ELH. Writing an integrative review. AORN J. 1998;67(4):877-80.

20. Stephenson MT, Witte K, Vaught C, Quick BL, Booth-Butterfield S, Patel D, et al. Using persuasive messages to encourage voluntary hearing protection among coal miners. J Safety Res. 2005;36(1):9-17.

21. Hong O, Ronis DL, Lusk SL, Kee GS. Efficacy of a computer-based hearing test and tailored hearing protection intervention. Int J Behav Med. 2006;13(4):304-14.

22. Trabeau M, Neitzel R, Meischke H, Daniell WE, Seixas NS. A comparison of "train-the-trainer" and expert training modalities for hearing protection use in construction. Am J Ind Med. 2008;51(2):130-7. 
23. Edelson J, Neitzel R, Meischke H, Daniell W, Sheppard L, Stover B, et al. Predictors of hearing protection use in construction workers. Ann Occup Hyg. 2009;53(6):605-15.

24. Stephenson MR, Shaw PB, Stephenson CM, Graydon PS. Hearing loss prevention for carpenters: part 2 - demonstration projects using individualized and group training. Noise Health. 2011;13(51):122-31.

25. Kim Y, Park J, Park M. Creating a culture of prevention in occupational safety and health practice. Saf Health Work. 2016;7(2):89-96

26. McCullang MC, Banerjee T, Cohen MA, Yang JJ. Effects of interventions on use of hearing protectors among farm operators: a randomized controlled trial. Int J Audiol. 2016; 55(supl 1):S3-S12.

27. Bramatti L, Morata TC, Marques JM. Ações educativas com enfoque positivo em programa de conservação auditiva e sua avaliação. Rev. CEFAC. 2008;10(3):398-408.

28. Ferreira LP, Caraça EB, Almeida ACB, Silva MAA. Assessoria fonoaudiológica: análise de um processo de construção entre o fonoaudiólogo e o teleoperador. Distúrb Comum. 2008;20(2):219-28.

29. Rocha CH, Santos LHD, Moreira RR, NevesLobo IF, Samelli AG. Verificação da efetividade de uma ação educativa sobre proteção auditiva para trabalhadores expostos a ruído. J Soc Bras Fonoaudiol. 2011;23(1):38-42.

30. Heupa AB, Gonçalves CGO, Albizu EJ, Dantas MR, Lacerda ABM, Lobato DCB. Programa de prevenção de perdas auditivas em pescador: perfil auditivo e ações educativas. Rev CEFAC. 2011;13(6):1009-16.

31. Sviech PS, Gonçalves CGO, Morata TC, Marques JM. Avaliação do conforto do protetor auditivo individual numa intervenção para prevenção de perdas auditivas. Rev CEFAC. 2013;15(5):1325-37.

32. Moreira AC, Gonçalves CGO. A eficiência de oficinas em ações educativas na saúde auditiva realizadas com trabalhadores expostos ao ruído. Rev CEFAC. 2014;16(3):723-31.

33. Samelli AG, Rocha CH, Theodósio P, Moreira RR, Neves-Lobo IF. Training on hearing protector insertion improves noise attenuation. CoDAS. 2015:27(6):514-19.

34. Cavalcante F, Ferrite S, Meira TC. Exposure to noise in the manufacturing industry in Brazil. Rev CEFAC. 2013;15(5):1364-70.

35. Masterson EA, Deddens JA, Themann CL, Bertke $\mathrm{S}$, Calvert GM. Trends in worker hearing loss by industry sector, 1981-2010. Am J Ind Med. 2015;58:392-401.

36. Neitzel R, Meischke H, Daniell WE, Trabeau M, Somers S, Seixas NS. Development and pilot test of hearing conservation training for construction workers. Am J Ind Medicine. 2008; 51:120-9.

37. Gonçalves CGO, Ribeiro JM. Análise dos programas de preservação auditiva em empresas do interior do Paraná. Tuiuti: Ciência e Cultura. 2013:46:12936.

38. Gonsalez CAG, Gonçalves CGO, Ribas A, Guida HL. O conhecimento e a percepção de supervisores de produção de uma indústria automobilística situada na região metropolitana de Curitiba sobre a saúde auditiva no trabalho. Conhec Interat. 2015;9(1):50-61.

39. Pender N, Walker S, Sechrist K, Frank-Stromborg M. Predicting health-promoting lifestyles in the workplace. Nurs Res. 1990;39(6):326-32.

40. Rosenstock IM. The health belief model and preventive health behavior. Health Educ Monogr. 1974;2:354-86.

41. Melamed S, Rabinowitz S, Feiner M, Weisberg E, Ribak J. Usefulness of the protection motivation theory in explaining hearing protection device use among male industrial workers. Health Psychol. 1996;15(3):209-15.

42. Vasconcellos EM. Educação popular e a atenção à saúde da família. $2^{a}$ ed. São Paulo: Hucitec; 2001

43. Vasconcellos LCF, Almeida CVB, Guedes DT. Vigilância em saúde do trabalhador: passos para uma pedagogia. Trab Educ Saúde. 2009;7(3):445-62.

44. Westphal MF. Promoção da saúde e prevenção de doenças. In: Campos GW. Tratado de saúde coletiva. São Paulo: Hucitec; 2006. p. 635-67.

45. Vilela RAG, Nobre Junior HB, Jackson Filho JM, Almeida IM. Ameaças à proteção do trabalho: o caso da segurança em máquinas e equipamentos. Rev Bras Saúde Ocup. 2015:40 (132):113-7.

46. Sbihi H, Teschke K, MacNab YC, Davies HW. An investigation of the adjustment of retrospective noise exposure for use of hearing protection devices. Ann Occup Hyg. 2010;54(3):329-39.

47. Arezes PM, Miguel AS. Assessing the use of hearing protection in industrial settings: a comparison between methods. Int J Ind Ergon. 2013;43(6):518-25.

48. Freire P. Pedagogia do oprimido. Rio de Janeiro: Edições Paz e Terra, 1970. 